\title{
Ultrasonic and Jet Aerosolization of Phospholipids and the Effects on Surface Activity
}

\author{
L. B. MARKS, R. H. NOTTER, ${ }^{(31)}$ G. OBERDORSTER, AND J. T. MCBRIDE
}

Departments of Pediatrics [L.B.M., R.H.N., J.T.M.] and of Radiation Biology and Biophysics [L.B.M., R.H.N., G.O.] University of Rochester School of Medicine Rochester, New York USA

\section{Summary}

Surface active aerosols were produced from aqueous dispersions of mixed lipids (CLL), extracted from bovine lung lavage. Particle size distributions were measured as a function of humidity for two types of aerosol generators: ultrasonic and jet. Lipid dispersions before aerosolization were prepared by sonication in an ice bath and by mechanical vortexing. Over a range of high humidity greater than 60-70\%, ultrasonic nebulization gave CLL aerosols with mass median aerodynamic diameters (MMAD) of $1.4 \pm 0.1$ $\mu \mathrm{m}$, compatible with predicted alveolar deposition fractions of $0.2-$ 0.3 according to current deposition models. For humidities of 30 95\%, jet nebulization gave MMAD values of 0.4-0.5 $\mu \mathrm{m}$, which have lower predicted alveolar deposition. The surface pressuretime $(\pi-t)$ adsorption characteristics at $35 \pm 2^{\circ} \mathrm{C}$ of CLL dispersions prepared initially by vortexing or sonication were not significantly affected by ultrasonic nebulization over a 1-2 h time period. In addition, the dynamic surface tension lowering of both kinds of CLL dispersion was not affected by ultrasonic nebulization (minimum surface tension $<1$ dyne $/ \mathrm{cm}$ at $37^{\circ} \mathrm{C}$ and $100 \%$ humidity). Current interest in the treatment of the respiratory distress syndrome (RDS) with exogenous surfactant replacement has focused largely on the delivery of surfactants to infants by tracheal instillation at birth. However, the ability to form multicomponent surfactant aerosols with appreciable alveolar deposition fractions and high surface activity may help to expand the utillity of replacement therapy to patients with aerated lungs.

\section{Abbreviations}

CLL, calf lung lipids

DPPC, dipalmitoyl phosphatidylcholine

DPPG, dipalmitoyl phosphatidylglycerol

egg-PG, egg phosphatidylglycerol

LPC, lyso-phosphatidylcholine

MMAD, mass median aerodynamic diameter

$\pi$, surface pressure

$\pi-t$, surface pressure-time

PC, phosphatidylcholine

RDS, respiratory distress syndrome

$S_{1}$, sonication in an ice bath.

TLC, thin layer chromatography

$V$, mechanical vortexing at room temperature

Much of the present interest in exogenous surfactant replacement therapy for neonatal RDS is centered on the delivery of surfactant mixtures to the respiratory system by direct airway instillation $(18,22,23)$. In animal model experiments for such therapy, the most effective time for surfactant instillation has been found to be before, or at least very close to, the onset of breathing (22). Current research (e.g., 10) also indicates that a single preventilatory dose of exogenous surfactant material can have an initial beneficial effect on lung function, which then decreases with time after instillation. The possibility thus exists that repeti- tive dose therapy might be beneficial in the treatment of RDS, and this in turn raises the question of what method might be most appropriate for the delivery of surfactants to aerated lungs.

Although tracheal instillation may be a preferred method for lungs in the initial stage of ventilation, a useful technique for surfactant delivery to aerated alveoli is by surface active aerosols. Studies by several groups of investigators, Brain et al. (2) and Watson et al. (26), have indicated that aerosolization gives a more uniform distribution of alveolar deposition than that found with intratracheal instillation of various kinds of particles.

The use of surfactant aerosols in RDS therapy has received attention in the past. As reviewed elsewhere $(18,19)$, aerosols of pure DPPC have been used in several studies on lung surfactant replacement over a decade ago. More recently, Ivey et al. (9) reported a limited clinical study with an aerosol of 9:1 DPPC:DPPG. But it is complex multicomponent mixtures of naturally derived lung surfactant material that have thus far proved most useful for RDS therapy in animal model instillation studies $(19,22,23)$, and aerosols of such mixtures have received little investigation. Ikegami et al. (8) studied the effects of natural lung surfactant aerosols on excised lung pressure-volume characteristics. However, detailed descriptions of the aerosols in terms of particle size distribution, humidity dependence, or intrinsic surface activity at physiologic conditions were not reported.

The present study is concerned with the aerosolization of dispersions of mixed lipids (CLL) extracted from bovine lung lavage. The CLL preparation studied has been shown to be effective in improving pulmonary function and gas exchange in premature lambs $(3,4,17)$. The primary focus of the work is to characterize multicomponent phospholipid aerosols in terms of surface activity and particle size distribution for nebulization by both ultrasonic and jet type aerosol generators. Ultrasonic nebulization is the technique that has generally been used in previous clinical studies with DPPC aerosols. A potential disadvantage of the ultrasonic nebulizer is that it subjects the solution to be aerosolized to significant vibrational energy. Phospholipid systems in aqueous dispersion can be sensitive to such effects (19), and it is possible that an ultrasonic nebulizer might alter the surface properties or biochemical composition of therapeutic surfactant mixtures. In addition to surface property effects, another variable studied is the humidity dependence of the particle size distribution of CLL aerosols by ultrasonic and jet nebulization. Humidity dependence is characterized because of its importance for particles entering the humid airways and its effect on alveolar deposition.

\section{MATERIALS AND METHODS}

Lipids. CLL were obtained by chloroform-methanol extraction of $0.15 \mathrm{M} \mathrm{NaCl}$ bovine lung lavage as described previously $(4,12$, 20). The lipids were analyzed by phosphorus determination and TLC to be $91 \%$ by weight phospholipid, with $73 \%$ phosphatidylcholine, $8 \%$ phosphatidylglycerol, $6.8 \%$ phosphatidylethanolamine, $5.3 \%$ phosphatidylinositol, $3.8 \%$ sphingomyelin and $3.1 \%$ phosphatidylserine. The CLL contained $6 \%$ cholesterol and cholesterol esters, and of order 1 weight $\%$ protein. 
The composition of CLL was re-analyzed by TLC after 1-h and 2-h exposures to ultrasonic nebulization to rule out the possibility of significant lipid hydrolysis with this method of aerosolization. TLC analysis with the solvent system of Touchstone et al. (25), and with the system chloroform, methanol, $7 \mathrm{M}$ ammonium hydroxide 60:35:5, showed no significant compositional changes in CLL after ultrasonic nebulization. Furthermore, to determine specific conversion of PC to LPC, $\left[{ }^{14} \mathrm{C}\right.$ dipalmitate-labeled DPPC was mixed with CLL and nebulized for $2 \mathrm{~h}$ in the closed chamber of the ultrasonic nebulizer. Conversion of PC to LPC was $\leqslant 2 \%$ after $2 \mathrm{~h}$ of continuous ultrasonic nebulization and reflux.

Aerosol generators. Although there are a number of different aerosol generator types, two which have been applied widely for clinical use because of their reliability and simplicity are ultrasonic and jet type nebulizers (13).

Ultrasonic nebulizer. These experiments used a Heyer Ultrasonic Nebulizer (USE 77) with an operating frequency of 2.5-2.8 megaHertz. An insert (medication cup) was used to minimize the volume of the solution to be nebulized. This cup was partially submerged in a liquid bath in the aerosol generator. In an ultrasonic nebulizer, vibrations are generated by a piezoelectric crystal at the convex base of the bath, and are transmitted through the bath to the solution. The ultrasonic vibrations cause the formation of capillary waves at the solution surface and these give rise to aerosol droplets. The capillary wavelength $(\lambda)$ between crests for an ultrasonic nebulizer is given by (11):

$$
\lambda=\left(8 \pi \gamma / \rho \mathrm{f}^{2}\right)^{1 / 3}
$$

where: $\gamma$, surface tension; $\rho$, density of the solution; and $f$, vibrational frequency. The capillary wavelength $(\lambda)$ in Equation (1) has been shown to have a strong association with the droplet size leaving the surface of the solution being nebulized (11). If surface tension approaches zero, Equation (1) predicts that capillary waves will not form. Although $\gamma$ does approach zero for lung surfactant films, this is only true under dynamic compression. In the absence of dynamic compression to monolayer collapse, $\gamma$ is non-zero for lung surfactant component films, and ultrasonic nebulization of CLL dispersions occurs readily as shown in "Results." For the ultrasonic nebulizer, the humidity of the aerosol leaving the generator is initially high, generally $>80 \%$.

Jet nebulizer. Jet experiments were done with a Dautrebande D-30 nebulizer. In a jet-type nebulizer, gas passes through a nozzle at high velocity. This causes a low pressure that entrains liquid droplets from the solution in the nebulizer reservoir. Many of these recruited (or primary) droplets are recycled back into the reservoir solution as they impact on structures within the nebulizer. For the D-30 jet nebulizer used in this study, a network of small capillary holes $(D=1-2 \mathrm{~mm}$ ) was present to cause the impaction and reflux of large aerosol droplets. Because of the complex geometry and flow patterns that affect particle size distributions from jet nebulizers, no generally applicable correlation is available for a priori prediction of aerosol size distribution (13). For jet type nebulizers, the humidity of the aerosol initially generated is low, of order $40-45 \%$.

Particle size distributions. The continuous flow system used to measure aerosol particle size distribution as a function of humidity is described in detail by Marks et al. (12). In brief, compressed filtered air was delivered to a given nebulizer to generate an aerosol stream. This aerosol was then mixed with a diluting air stream of variable humidity. The humidity of the final aerosol stream was a function of the flow rates and humidities of the two air streams. The final aerosol stream was allowed to equilibrate at a given humidity by passage through a tubing system, which allowed aerosol residence times of up to $15 \mathrm{sec}$. This is several orders of magnitude larger than the times found necessary for size equilibration of saline aerosols at a given humidity $(1,6)$.

After equilibration, the particle size distribution of the aerosol was measured with a seven stage cascade impactor as described by Mercer et al. (14). In this type of apparatus, the aerosol flows through small holes past a series of stages. The air passes around the stages but inertial effects cause the particles to impact on glass plates that sit on each stage. The orifice size for flow between each stage of the impactor decreases continuously, and the aerosol particles impact on the stages according to their aerodynamic diameter. The critical orifice at the last stage of the impactor determines the flow rate through the system.

In the experiments here, the flow rate through the impactor was $0.92 \mathrm{liter} / \mathrm{min}$, which was provided by a piston pump (Terracon Corp, Type $\mathbf{R}$ 506). The impactor was designed to measure particles with aerodynamic diameters ranging from 0.166-5.3 $\mu \mathrm{m}$. A number of other kinds of diameter such as geometric and count median diameters may be defined for an aerosol in addition to the aerodynamic diameter (13); however, for clinical purposes, the aerodynamic diameter is probably the most useful because it relates directly to the pulmonary deposition pattern of the aerosol. For the experiments here, the aerodynamic diameter measured is the activity median aerodynamic diameter, equivalent to the MMAD.

The mass of "dry" material ( $\mathrm{NaCl}$ and $\mathrm{CLL})$ deposited on each impactor stage was measured from the activity of a [ ${ }^{99 \mathrm{~m}} \mathrm{Tc}$ ] label which was used to mark the aqueous phase. This label then remained with the dry particles on the impactor stages after water evaporation. Since the Tc activity of the solution in the aerosol generator was known, the mass of $\mathrm{NaCl}$ (or CLL) on a given stage could be found as:

$$
\begin{aligned}
& \text { Mass of } \mathrm{NaCl} \text { on stage } \mathrm{X} \\
&= {\left[\frac{\mathrm{Tc} \text { activity, stage } \mathrm{X}}{\mathrm{Tc} \text { activity per volume in nebulizer solution }}\right] \times } \\
& \cdot\left[\frac{\mathrm{Mass} \text { of } \mathrm{NaCl} \text { in solution }}{\text { Volume of solution }}\right]
\end{aligned}
$$

Because the CLL being nebulized was a highly multicomponent amphipathic mixture, it was necessary to verify that it was nebulized proportionally with the aqueous phase by the aerosol generator. This was studied in a double label experiment with $\left[{ }^{14} \mathrm{C}\right]$ DPPC, which is the major component of CLL (and also of lung surfactant). The $\left[{ }^{14} \mathrm{C}\right]$ DPPC was mixed with CLL in chloroform, where the lipids are totally soluble. The chloroform was then evaporated under nitrogen, and the $\left[{ }^{14} \mathrm{C}\right]$ labeled lipids dispersed in $0.15 \mathrm{M} \mathrm{NaCl}$ by $\mathrm{S}_{i}$ or $\mathrm{V}$. The aqueous dispersions were then labeled with [9m $\mathrm{Tc}$ ], and nebulized in the jet and ultrasonic generators. These double-label experiments reported in detail by Marks et al. (12) indicated that neither CLL nor $\mathrm{NaCl}$ were aerosolized preferentially, and that lipid aerosol particle size distributions could be accurately measured by an aqueous phase radiolabel [ $\left.{ }^{99 m} \mathrm{Tc}\right]$.

Adsorption. $\pi-\mathrm{t}$ adsorption characteristics were measured at $35 \pm 2{ }^{\circ} \mathrm{C}$ as described previously by Notter et al. (20). For experiments involving the adsorption of lipid dispersions after exposure to ultrasonic nebulization, the procedure was as follows. Initial dispersion of $5 \mathrm{mg}$ of CLL per $\mathrm{ml}$ of $0.15 \mathrm{M} \mathrm{NaCl}$ was accomplished by $\mathrm{V}$ at room temperature $\left(22-23^{\circ} \mathrm{C}\right)$ or by $\mathrm{S}_{\mathrm{i}}$. These dispersions were then placed in the Heyer Generator and the output port was capped so that the nebulization compartment was closed. Nebulization was then started; the resultant aerosol droplets coalesced and refluxed back into the bulk solution. Because of the convection currents from the vigorous agitation present in the ultrasonic nebulization process, the bulk solution in the nebulizer cup was well-mixed. After a given time of continuous nebulization, $1 \mathrm{ml}$ aliquots were removed from the cup solution. In $\pi-\mathrm{t}$ adsorption experiments, each $1-\mathrm{ml}$ aliquot was injected beneath the surface of a stirred subphase of $70 \mathrm{ml}$ of $0.15 \mathrm{M} \mathrm{NaCl}$, and $\pi$ was then monitored as a function of time by measurement of the force on a sandblasted platinum slide dipped in the surface (20).

Adsorption experiments of this type were done for CLL dispersions subjected to continuous aerosolization and reflux in the closed nebulizer cup for times of $5 \mathrm{~min}$ to $2 \mathrm{hs}$. For this relatively long time scale of nebulization and reflux ( $\min$ to $h$ ), it is reasonable to assume that the final nebulizer cup solution approximates a collection of aerosol droplets, i.e., that the majority of the CLL 
dispersion has gone through the actual nebulization process one or more times.

Dynamic surface tension lowering. The effect of nebulization on the magnitude of surface tension lowering achieved by CLL dispersions under dynamic compression was measured at $37^{\circ} \mathrm{C}$ and $100 \%$ humidity with an oscillating bubble apparatus described in detail by Enhorning (5). Experiments involving dynamic surface pressure were done on CLL dispersions $(5 \mathrm{mg} / \mathrm{ml})$ subjected to ultrasonic nebulization for various times as described above under adsorption studies.

\section{RESULTS}

\section{CLL_SALINE AEROSOL PARTICLE SIZE DISTRIBUTIONS}

Ultrasonic nebulization. The particle size distributions for aerosols produced by ultrasonic nebulization of lipid dispersions are given as a function of humidity in Table 1 . Results are presented in terms of the MMAD and the geometric standard deviation, $\sigma_{\mathrm{g}}$. A major feature of the data in Table 1 is that the MMAD of lipid aerosols produced by ultrasonic nebulization is very stable at about $1.4 \mu \mathrm{m}$ over a range of high humidity (i.e., relative humidity $>60-70 \%$ ). This MMAD stability for lipid aerosols (27) is quite different from the behavior of pure saline aerosols, which show a continuous decrease in median particle size as humidity decreases from $100 \%$ (24). Possible biophysical mechanisms for CLL aerosol stability are discussed in detail by Marks et al. (12). For the present paper, the existence of MMAD values of $1.3-1.5 \mu \mathrm{m}$ for relative humidity $>60-70 \%$ is most important in terms of its ramifications for pulmonary deposition as discussed later.

Jet nebulization. Particle size distribution results for CLL nebulized by the jet-generator are given in Table 2 for the cases of initial dispersion by $S_{i}$ and $V$. The results show that jet nebulized CLL particles are essentially constant in diameter as humidity is varied over the range of $30-95 \%$. Equilibration times of $0.08-14$ sec had no effect in terms of particle size measurements. The constant MMAD behavior found for CLL jet nebulization is again different from that found by jet nebulization of pure saline

Table 1. Particle size distributions of aerosols produced from CLL dispersions by ultrasonic nebulization

\begin{tabular}{clcc}
\hline $\begin{array}{c}\text { Relative } \\
\text { humidity } \\
(\%)\end{array}$ & $\begin{array}{c}\text { Mass median } \\
\text { aerodynamic } \\
\text { diameter }(\mu \mathrm{m})\end{array}$ & $\begin{array}{c}\text { Geometric } \\
\text { standard } \\
\text { deviation' } \\
\sigma_{\mathrm{g}}\end{array}$ & $\begin{array}{c}\text { CLL } \\
\text { initially } \\
\text { dispersed } \\
\text { by }\end{array}$ \\
\hline$\geq 99$ & $1.48^{2}(n=2)$ & 2.0 & $\mathbf{S}_{\mathrm{i}}$ \\
95 & $1.33(2)$ & 1.8 & $\mathrm{~S}_{\mathrm{i}}$ \\
$87-90$ & $1.33(2)$ & 1.8 & $\mathrm{~S}_{\mathrm{i}}$ \\
83 & $1.45(1)$ & 1.9 & $\mathrm{~S}_{\mathrm{i}}$ \\
$77-80$ & $1.36(3)$ & 1.8 & $\mathrm{~S}_{\mathrm{i}}$ \\
$71-75$ & $1.48(2)$ & 2.0 & $\mathrm{~S}_{\mathrm{i}}$ \\
$60-63$ & $1.32(3)$ & 2.0 & $\mathrm{~S}_{\mathrm{i}}$ \\
$55-56$ & $0.89(2)$ & 1.9 & $\mathrm{~S}_{\mathrm{i}}$ \\
45 & $0.54(1)$ & 1.7 & $\mathrm{~S}_{\mathrm{i}}$ \\
38 & $0.38(1)$ & 1.8 & $\mathrm{~S}_{\mathrm{i}}$ \\
95 & $1.38(n=2)$ & 1.8 & $\mathrm{~V}$ \\
$90-91$ & $1.34(2)$ & 1.8 & $\mathrm{~V}$ \\
$80-86$ & $1.39(4)$ & 1.8 & $\mathrm{~V}$ \\
73 & $1.44(2)$ & 1.7 & $\mathrm{~V}$ \\
68 & $0.70(1)$ & 1.7 & $\mathrm{~V}$ \\
$59-62$ & $0.72(3)$ & 1.9 & $\mathrm{~V}$ \\
$25-31$ & $0.49(4)$ & 1.7 & $\mathrm{~V}$ \\
\hline
\end{tabular}

${ }^{1}$ For a log-normal aerosol size distribution, $D_{50}$ and $D_{84}$ can be defined as the diameter at which $50 \%$ and $84 \%$, respectively, of the total mass of aerosol particles can be accounted for by particles of diameter $D<D_{60}$ or $\mathrm{D}<\mathrm{D}_{84}$. The geometric standard deviation $\sigma_{8}$ is defined as $\mathrm{D}_{\mathbf{8 4}} / \mathrm{D}_{\mathbf{5 0}}$.

${ }^{2}$ Values expressed are averages of $n$ experiments (in parentheses) over the humidity range given. Because of mechanical factors it was not possible to duplicate a given humidity exactly in repetitive experiments.
Table 2. Particle size distributions of aerosols produced from $C L L$ dispersions by jet nebulization

\begin{tabular}{clcc}
\hline $\begin{array}{c}\text { Relative } \\
\text { humidity } \\
(\%)\end{array}$ & $\begin{array}{c}\text { Mass median } \\
\text { aerodynamic } \\
\text { diameter }(\mu \mathrm{m})\end{array}$ & $\begin{array}{c}\text { Geometric } \\
\text { standard } \\
\text { deviation } \\
\sigma_{g}\end{array}$ & $\begin{array}{c}\text { CLL } \\
\text { initially } \\
\text { dispersed } \\
\text { by }\end{array}$ \\
\hline$\geq 95$ & $0.43^{\prime}(n=3)$ & $1.5^{1}$ & $\mathrm{~S}_{\mathrm{i}}$ \\
$90-91$ & $0.46(2)$ & 1.4 & $\mathrm{~S}_{\mathrm{i}}$ \\
$78-80$ & $0.48(2)$ & 1.5 & $\mathrm{~S}_{\mathrm{i}}$ \\
$70-74$ & $0.43(4)$ & 1.5 & $\mathrm{~S}_{\mathrm{i}}$ \\
$60-66$ & $0.45(3)$ & 1.6 & $\mathrm{~S}_{\mathrm{i}}$ \\
52 & $0.42(1)$ & 1.7 & $\mathrm{~S}_{\mathrm{i}}$ \\
$36-38$ & $0.44(2)$ & 1.8 & $\mathrm{~S}_{\mathrm{i}}$ \\
30 & $0.40(1)$ & 1.7 & $\mathrm{~S}_{\mathrm{i}}$ \\
$\geq 95$ & $0.41(2)$ & 1.5 & $\mathrm{~V}$ \\
60 & $0.45(4)$ & 1.5 & $\mathrm{~V}$ \\
$30-35$ & $0.39(2)$ & 1.5 & $\mathrm{~V}$ \\
\hline
\end{tabular}

${ }^{1}$ Values for MMAD and $\sigma_{\mathrm{g}}$ are averages of $\boldsymbol{n}$ experiments (in parentheses) within the stated humidity range.

Table 3. Dynamic surface tension lowering of lipids exposed to ultrasonic nebulization

\begin{tabular}{cccc}
$\begin{array}{c}\text { Time in } \\
\text { nebulizer } \\
\text { (min) }\end{array}$ & $\begin{array}{c}\text { Minimum } \\
\text { surface } \\
\text { tension } \\
\text { (dyne/cm) }\end{array}$ & $\begin{array}{c}\text { Maximum } \\
\text { surface } \\
\text { tension } \\
\text { (dyne/cm) }\end{array}$ & $\begin{array}{c}\text { Initial } \\
\text { dispersion } \\
\text { method }\end{array}$ \\
\hline 0 & $<1$ & 30 & $\mathbf{V}$ \\
5 & $<1$ & 34 & $\mathbf{V}$ \\
10 & $<1$ & 33 & $\mathbf{V}$ \\
30 & $<1$ & 34 & $\mathbf{V}$ \\
60 & $<1$ & 34 & $\mathrm{~V}$ \\
0 & $<1$ & 33 & $\mathrm{~S}_{\mathbf{i}}$ \\
5 & $<1$ & 34 & $\mathrm{~S}_{\mathrm{i}}$ \\
10 & $<1$ & 33 & $\mathrm{~S}_{\mathrm{i}}$ \\
30 & $<1$ & 32 & $\mathrm{~S}_{\mathrm{i}}$ \\
60 & $<1$ & 34 & $\mathrm{~S}_{\mathrm{i}}$ \\
\hline
\end{tabular}

${ }^{1}$ Dynamic surface tension lowering measured at $37^{\circ} \mathrm{C}$ and $100 \%$ humidity with oscillating bubble. Cycle rate was $20 \mathrm{cycles} / \mathrm{min}$. Measurements made on $20 \mu \mathrm{L}$ aliquots $(5 \mathrm{mg} \mathrm{CLL} / \mathrm{ml}$ ) subjected to ultrasonic nebulization in Heyer generator for various times. See text for details.

solutions, which typically show a rapid rise in particle size when humidity reaches a value called the deliquescent humidity (21). Possible mechanistic explanations for the lack of deliquescence found for CLL aerosols from the jet nebulizer are discussed by Marks et al. (12).

Effects of nebulization on surface activity. The question of whether ultrasonic nebulization changes the surface properties of CLL mixtures was addressed by looking at the dynamic surface tension lowering and adsorption characteristics of CLL dispersions after they had been subjected to ultrasonic nebulization for various periods of time (see "Materials and Methods").

The dynamic surface tension lowering characteristics of CLL dispersions were measured at $37^{\circ} \mathrm{C}$ and $100 \%$ humidity in an oscillating bubble apparatus described by Enhorning (5). The results presented in Table 3 are expressed as minimum and maximum surface tensions of 20- $\mu$ liter aliquots of CLL dispersions (initially dispersed by $\mathbf{V}$ or $\mathrm{S}_{\mathrm{i}}$ ) compressed and expanded between $100 \%$ area and $50 \%$ area at a rate of $20 \mathrm{cycles} / \mathrm{min}$ in the oscillating bubble. Initial dispersion concentration was $5 \mathrm{mg} \mathrm{CLL} / \mathrm{ml}$. The time values in Table 3 represent the length of time the CLL dispersions were subjected to continuous ultrasonic nebulization (and reflux) in the cup of the Heyer generator when an aliquot was taken for surface tension measurement. As shown in Table 3, ultrasonic nebulization for times as long as $1 \mathrm{~h}$ did not change the minimum surface tension values achieved upon dynamic compression of CLL dispersions. 
The $\pi-t$ adsorption behavior of vortexed CLL dispersions subjected to ultrasonic nebulization for various times is shown in Figure 1. The results indicate that the adsorption of vortexed CLL dispersions is not significantly affected by ultrasonic nebulization for times as long as $\mathbf{2 ~} \mathrm{h}$. This is true in terms of both the surface pressure magnitude achieved and its time dependence. The lack of effect of ultrasonic nebulization on the adsorption of vortexed CLL dispersions is critical because such dispersions have been shown to improve lung function in premature lambs $(3,4,17)$, as noted earlier.

The effect of ultrasonic nebulization on the adsorption characteristics of sonicated CLL dispersions was also studied. The $\pi-$ $t$ behavior of CLL $\left(S_{i}\right)$ dispersions in Figure 2 shows that the mixture adsorption properties are not significantly affected by ultrasonic nebulization for times as long as $1 \mathrm{~h}$. In all cases in Figure 2, the $\pi-t$ adsorption of $\operatorname{CLL}\left(S_{i}\right)$ dispersions to surface pressure values of 45 dynes $/ \mathrm{cm}$ is rapid, and occurs over a shorter time-scale than that found for vortexed CLL dispersions at the dilute lipid concentration used $(0.07 \mathrm{mg} / \mathrm{ml})$. The $\pi-t$ behavior in Figure $2 \mathrm{~A}$ before nebulization is the same as that found previously by Notter $e t$ al. (20) for sonicated dispersions of another CLL preparation. The results in Figures $2 \mathrm{~B}$ and $2 \mathrm{C}$ show that this behavior persists after ultrasonic nebulization (28).

\section{DISCUSSION}

The finding that ultrasonic nebulization does not adversely affect the surface tension lowering behavior of CLL dispersions is
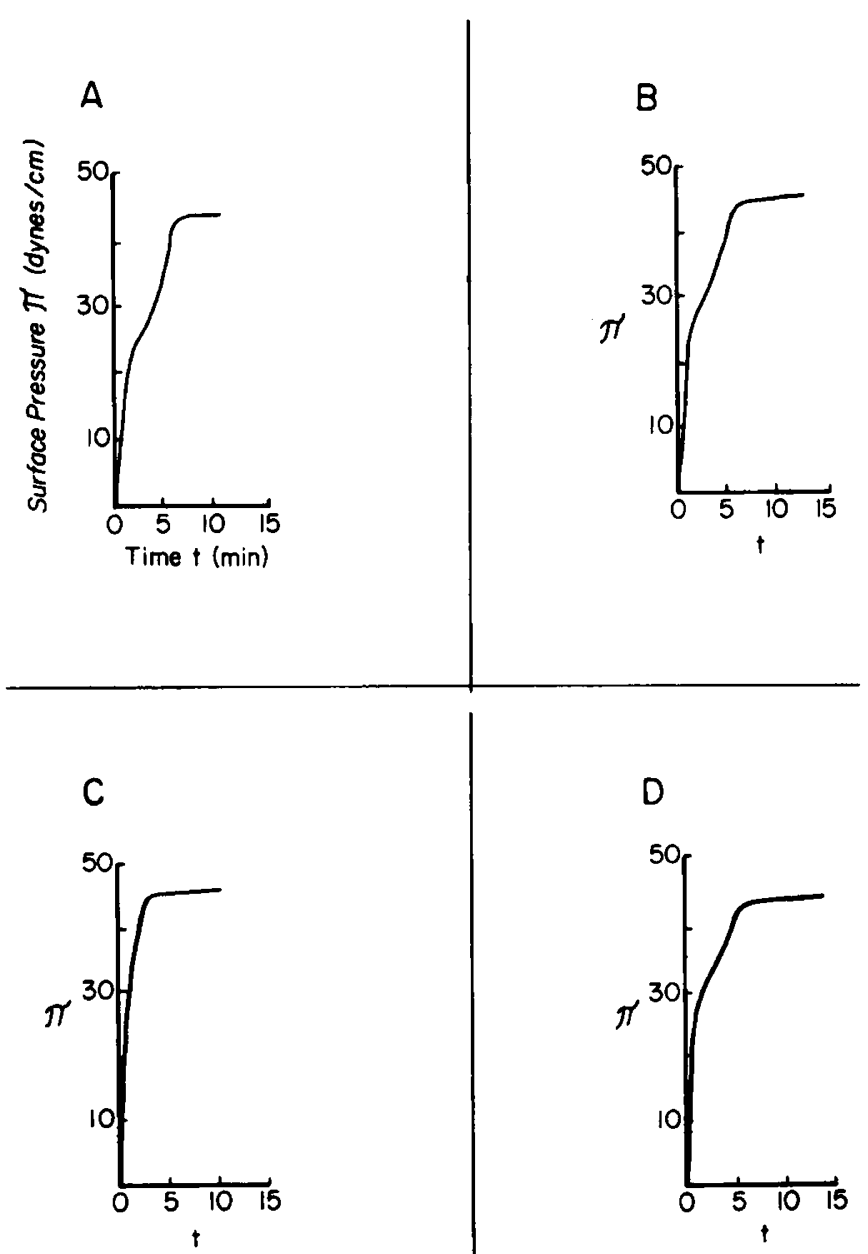

Fig. 1. Surface pressure-time $(\pi-t)$ adsorption of vortexed lipid dispersions subjected to ultrasonic nebulization. See "Materials and Methods" for details. (A) CLL (V), initial; (B) CLL (V) $30 \mathrm{~min}$ in nebulizer; (C) CLL (V), $60 \mathrm{~min}$ in nebulizer; and (D) CLL (V), $120 \mathrm{~min}$ in nebulizer. See text for details.
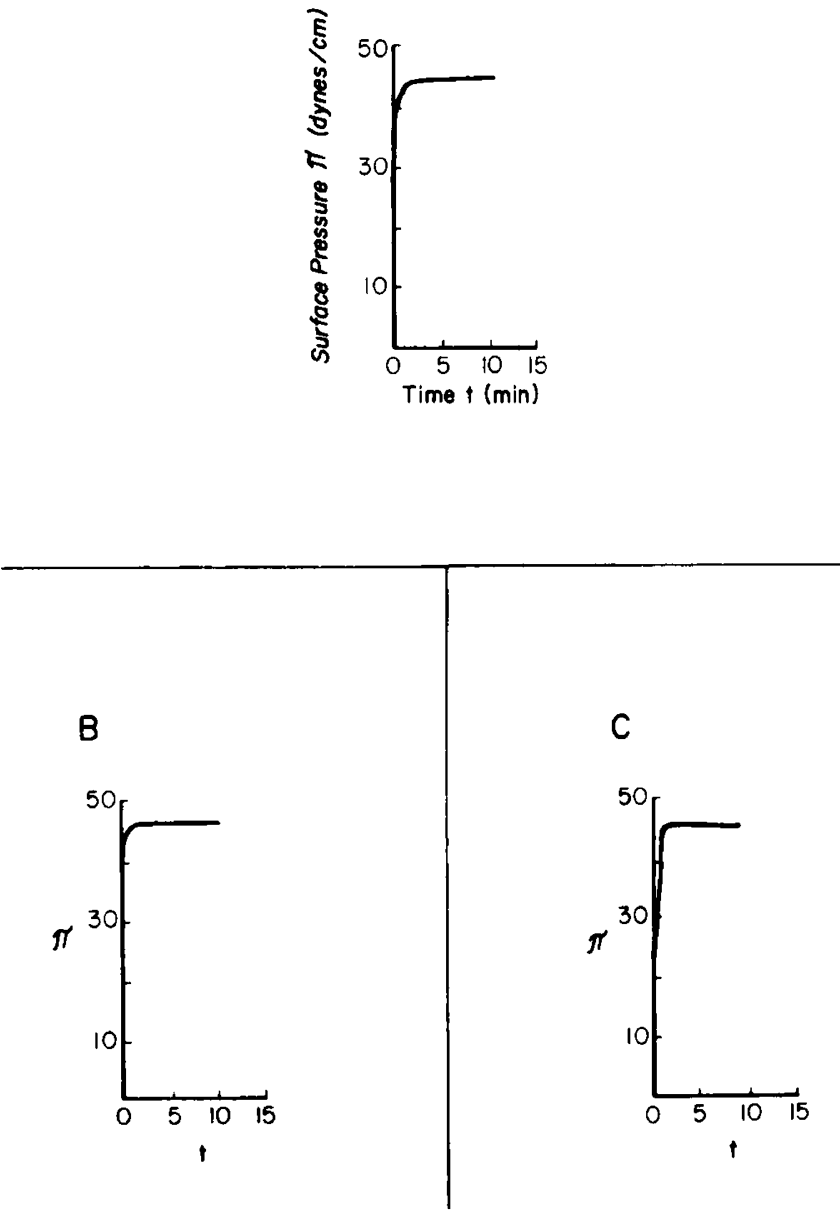

Fig. 2. Adsorption of sonicated lipid dispersions subjected to ultrasonic nebulization. See "Materials and Methods" for details. $(A) \operatorname{CLL}\left(\mathrm{S}_{\mathrm{i}}\right)$, initial; $(B)$ CLL $\left(\mathrm{S}_{\mathrm{i}}\right), 10 \mathrm{~min}$ in nebulizer; and $(C) \mathrm{CLL}\left(\mathrm{S}_{\mathrm{i}}\right), 60 \mathrm{~min}$ in nebulizer.

consistent with previous work (20), which showed that the adsorption of this kind of naturally derived lipid mixture is less sensitive to heating effects than phospholipid mixtures from non-pulmonary sources (e.g., DPPC-egg PG). The results of Figures 1 and 2 show that the adsorption of CLL dispersions is also relatively unaffected by exposure to a high ultrasonic energy input in the Heyer nebulizer. In addition, the data in Table 3 show that CLL dispersions are also not significantly affected in terms of dynamic surface tension lowering ability when subjected to ultrasonic nebulization.

For any surface active mixture of potential use in inhalation therapy for RDS, the pulmonary deposition characteristics of the aerosol form must be considered. The literature concerning aerosol deposition in the pulmonary system is complex, and a multitude of experimental studies and empirical correlations are available. Many of these correlations are discussed by Mercer (13), including the ICRP Task Group on Lung Dynamics model (7). According to this model, alveolar deposition fraction (including the respiratory bronchioles) peaks at about 0.3 for an aerodynamic diameter of $2 \mu \mathrm{m}$, and is still above 0.2 for diameters of 1 or $3 \mu \mathrm{m}$. Consequently, CLL aerosols produced by ultrasonic nebulization with MMAD $=1.4 \pm 0.1$ and $\sigma_{\mathrm{g}}=1.7-1.9$ should yield alveolar depositions of more than $20 \%$ at physiologic humidity. Because of the stability of aerosol size distribution for relative humidity $>70 \%$ precise control of humidity would not be required in lipid aerosol delivery sysems used in a clinical setting. Humidity must be maintained at values above about $60 \%$, however, or the aerosol droplets will evaporate to dry particles which will not easily grow back to droplets (Table 1).

The consumption of solution with the Heyer ultrasonic nebu- 
lizer was found to be in the range $0.05-0.3 \mathrm{ml} / \mathrm{min}$ for air flow rates of $0.3-7.5 \mathrm{liter} / \mathrm{min}$. These values are compatible with delivery of sufficient surface active material to cover the alveolar interface with a tight packed monolayer in a time period practical in an intensive care nursery. For example, if a value of $8 \mathrm{ml} / \mathrm{kg} /$ breath is assumed for an infant (16), a typical minute volume at $30-50$ breaths/min is just below the lower range of air flow rates studied. For a solution consumption of $0.05 \mathrm{ml} / \mathrm{min}$, a CLL solution concentration of $10 \mathrm{mg} / \mathrm{ml}$, and an alveolar deposition fraction of 0.2 , this gives $0.1 \mathrm{mg}$ CLL delivered to alveoli per min. If a gas exchange surface area of $1 \mathrm{~m}^{2} / \mathrm{kg}$ is assumed, it takes 3.1 $\mathrm{mg} / \mathrm{kg}$ to cover the alveolar surface with a close packed monolayer of CLL (molecular weight assumed as 750) occupying $40 \AA^{2} /$ molecule. This dose could be delivered to a 1-kg infant in approximately $30 \mathrm{~min}(29)$.

In terms of the evaluation of surface active replacement mixtures for possible use in RDS therapy, research in the past decade has dealt most thoroughly with pulmonary delivery by direct tracheal instillation $(22,23)$. A wide variety of animal model studies have demonstrated improved lung function following the instillation of multicomponent surfactant mixtures at or before the first breath, and this is clearly the indicated therapeutic approach when feasible. However, it is also true that pre-ventilatory tracheal instillation will not be possible for all patients suffering from a primary or secondary surfactant deficiency. The relative advantages and disadvantages of multicomponent phospholipid aerosols compared to tracheal instillation therapy for such patients have not yet received a sufficiently comprehensive evaluation in terms of animal models studied, or mixtures and aerosol generation techniques used. Previous aerosol work has concentrated largely on nebulized DPPC, and the few studies involving multicomponent phospholipid mixtures $(8,9)$ have not included detailed characterization of intrinsic aerosol surface activity at physiologic conditions. The present results for ultrasonically nebulized CLL suggest that it is one candidate (of perhaps many) which might profitably be studied further in animal models of RDS.

The MMAD values of 0.4-0.5 $\mu \mathrm{m}$ found for jet produced CLL aerosols in Table 2 are not as optimal in terms of alveolar deposition as the larger values found in ultrasonic nebulization. The ICRP model $(7,13)$ does predict significant alveolar deposition fractions for $0.5 \mu \mathrm{m}$ particles, but the magnitude is not as large as the fraction predicted for $1.5 \mu \mathrm{m}$ particles. In addition, a jet-type nebulizer has several potential disadvantages compared to an ultrasonic nebulizer for delivery of surface active aerosols to infants. The jet nebulizer uses a relatively large amount of solution (about $0.2 \mathrm{ml} / \mathrm{min}$ ) because of the large continuous air flow rate needed for jet nebulization. By contrast, an ultrasonic nebulizer can easily be used as a demand system: aerosol can be drawn off from the generator chamber only as required by any given ventilator setting.

The jet nebulizer particle size results in Table 2 are perhaps most important because of the hysteresis they show in the humidity dependence of the phospholipid aerosols. A discussed in detail by Marks et al. (12), the size distribution data in Tables 1 and 2 can be combined in a single plot of MMAD vs relative humidity. One feature resulting from such analysis is that CLL aerosols exposed to low humidity $(<50 \%)$ will evaporate from droplets to dry particles, as noted above. If these particles are subsequently exposed to high humidity, they do not readily grow back to droplets (Table 2). The ability to form stable aerosols of phospholipid particles (as opposed to droplets) may prove useful in surfactant replacement applications involving mixtures such as 7.3 DPPC:PG, which has been used in "dry" powder form by Morley et al. (15).

In summary, these experiments have shown that it is feasible to form multicomponent aerosols of surface active phospholipids by ultrasonic and jet nebulization. In terms of surface property effects, it was found that the process of ultrasonic nebulization did not change the dynamic surface tension lowering effectiveness of CLL dispersions at physiologic temperature and humidity. Moreover, the character of the $\pi-t$ adsorption isotherm for CLL dispersions prepared by mechanical vortexing or sonication was not significantly changed by ultrasonic nebulization for times as long as two hours. The particle size distributions found for both aerosol generation techniques at high humidity (relative humidity $>60-70 \%$ ) are compatible with some alveolar deposition. However, the alveolar deposition fraction of $0.2-0.3$ predicted for CLL aerosols of MMAD $1.4 \pm 0.1 \mu \mathrm{m}$ produced from ultrasonic nebulization is greater than that predicted for the smaller particles of MMAD = 0.4-0.5 $\mu \mathrm{m}$ produced from jet nebulization. It would appear possible to produce surface active aerosols of multicomponent lipid mixtures that have potential for practical applications to neonatal RDS therapy in aerated lungs.

\section{REFERENCES AND NOTES}

1. Bell, K. A. and Ho, A. T.: Growth rate measurements of hygroscopic aerosols under conditions simulating the respiratory tract. J. Aerosol Sci., 12: 247 (1981).

2. Brain, J. D., Knudsen, D. E., Sorokin, S. P., and Davis, M. A.: Pulmonary distribution of particles given by intratracheal instillation or by aerosol inhalation. Environ. Res., 11: 13 (1976).

3. Egan, E. A., Notter, R. H., and Shapiro, D. L.: Natural and artificial surfactant replacement in premature lambs. Pediatr. Res. (Abstract), I6: 348 (1982).

4. Egan, E. A., Notter, R. H., Kwong, M. S., and Shapiro, D. L.: Natural and artificial lung surfactant replacement in premature lambs. J. Appl. Physiol., (In press) 1983.

5. Enhorning, G.: Pulsating bubble technique for evaluating pulmonary surfactant. J. Appl. Physiol., 43: 198 (1977).

6. Golli, S. E., Bricard, J., Turpin, P. Y., and Treiner, C.: The evaporation of saline droplets. J. Aerosol Sci., 5: 273 (1974).

7. ICRP Publication 30, part 1: Limits for intakes of radionucleotides by workers. pp. 23-29 (Pergamon Press, Oxford, 1979).

8. Ikegami, M., Hesterberg, T., Nozaki, M., and Adams, F. H.: Restoration of lung pressure-volume characteristics with surfactant: comparison of nebulization versus instillation and natural versus synthetic surfactant. Pediatr. Res., 11: 178 (1978).

9. Ivey, H., Roth, S., and Kattwinkel, J.: Nebulization of sonicated phospholipids for treatment of respiratory distress syndrome of infants. Pediatr. Res. (Abstract), 12: 573 (1978).

10. Jobe, A., Ikegami, M., Glatz, T., Yoshida, Y., Diakomanolis, E., and Padbury, J.: Duration and characteristics of treatment of premature lambs with natural surfactant. J. Clin. Invest., 67: 370 (1981).

11. Lang, R. J.: Ultrasonic atomization of liquids. J. Acoust. Soc. Am., 34: 6 (1962)

12. Marks, L. B., Oberdorster, G., and Notter, R. H.: Generation and characterization of aerosols of dispersed surface active phospholipids by ultrasonic and jet nebulization. J. Aerosol Sci., (In press) 1983.

13. Mercer, T.: Aerosol technology in hazard evaluation. (Academic Press, New York, 1973).

14. Mercer, T. T., Tillery, M. I., and Newton, G. J.: A multistage, low flow rate cascade impactor. J. Aerosol Sci., J: (1970).

15. Morley C. J., Miller, N., Bangham, A. D., and Davis, J. A.: Dry artificial lung surfactant and its effect on very premature babies. Lancet, 1: 64 (1981)

16. Nelson, W.: Textbook of Pediatrics, 9th Edition. (Eds.) W. Nelson, V. Vaughan, R. J. McKay.: (W.B. Saunders Co., Philadelphia, 1969).

17. Notter, R. H., Egan, E. A., and Shapiro, D. L.: Effect of phospholipid adsorption facility on lung surfactant replacement therapy. Federation Proceedings (Abstract), 41: 1125 (1982).

18. Notter, R. H. and Morrow, P. E.: Pulmonary surfactant: a surface chemistry viewpoint. Ann. Biomed. Engr., 3: 119 (1975).

19. Notter, R. H. and Shapiro, D. L.: Lung surfactant in an era of replacement therapy. Pediatrics, 68: 781 (1981).

20. Notter, R. H., Smith, S., Taubold, R. D., and Finkelstein, J., N.: Path dependence of adsorption behavior of mixtures containing dipalmitoyl phosphatidylcholine. Pediatr. Res., 16: 515 (1982).

21. Pruppacher, U. R. and Klett, J. D.: Microphysics of clouds and precipitation. (D. Reidel Publishing Co., Boston, 1978).

22. Robertson, B.: Surfactant substitution: experimental models and clinical applications. Lung, 158: 57 (1980).

23. Robertson, B.: Neonatal pulmonary mechanics and morphology after experimental therapeutic regimens. In: E. M. Scarpelli, (Ed.): Reviews in Perinatal Medicine. Vol. 4, p. 337 (Raven Press, New York, 1981).

24. Tang, I. N., Munkelwitz, A. R., and Davis, J. G.: Aerosol Growth Studies. II. Preparation and growth measurements of monodisperse salt aerosols. J. Aerosol Sci., 8: 149 (1977).

25. Touchstone, J. C., Chen, J. C., and Beaver, K. M. Improved separation of phospholipids in thin layer chromatography. Lipids 15: 61 (1980).

26. Watson, J. A., Spritzer, A. A., Auld, J. A., and Guethoff, M. A. Deposition and clearance following inhalation and intra-tracheal injection of particles. Arch. Environ. Health, 9: 51 (1969).

27. Because of the stability found for CLL containing aerosols above $R H=60-70$ percent, the residence time in the aerosol flow system was varied from 0.5 seconds to 15 seconds to check for possible non-equilibrium effects. Over this residence time range, no change in particle size distribution was observed.

28. There is a slight slowing in adsorption rate in Figure $2 \mathrm{C}$ (after 60 minutes in nebulizer) compared to Figure $2 \mathrm{~A}$ (initial) and 2B (after 10 minutes in 
nebulizer). However, the adsorption rate in Figure 2C is still more rapid than that of vortexed CLL dispersions in Figure 1 for the uniform final dispersion concentration (5 mg CLL/71 ml subphase) used (see "Materials and Methods"). This dilute concentration was chosen because it allows a discrimination between the adsorption isotherms of CLL $\left(S_{i}\right)$ and CLL $(V)$. At higher concentrations ( $\geq 0.25 \mathrm{mg} / \mathrm{ml}$ ) vortexed CLL dispersions adsorb as rapidly as the sonicated dispersions in Figure 2.

29. If the assumption is made that all of the aerosolized solution (a total of $1.5 \mathrm{ml}$ in 30 minutes containing $0.15 \mathrm{M} \mathrm{NaCl}$ ) is retained by the infant, this would result in a sodium load of about $0.25 \mathrm{mEq}$. Note that the surfactant dose calculations here are for material which is actually deposited at the alveolar level ( $20 \%$ of inhaled amount). In animal studies of surfactant replacement $(22,23)$, typical tracheal instillation doses are 5-10 times larger than in this example calculation. However, a significant percentage of instilled surfactant probably does not reach the alveolar interface.

30. This paper is based on work performed under Contract No. DE-ACO276EV03490 with the U.S. Department of Energy at the University of Rochester Department of Radiation Biology and Biophysics and has been assigned Report No. UR-3490-2213. This work was also supported in part by NIH grants HL-25170 and K04-HL-00945. L. Marks was the recipient of a summer research fellowship at the University of Rochester School of Medicine (HHS grant HL-07496). The assistance of Dr. Richard Taubold with surface property measurements is gratefully acknowledged.

31. Requests for reprints should be addressed to: R. H. Notter, Department of Pediatrics, Division of Neonatology, University of Rochester School of Medicine, Rochester, New York 14642.

32. Received for publication October $22,1982$.

33. Accepted for publication March 9, 1983.

\title{
Pseudomonas-Infected Cystic Fibrosis Patient Sputum Inhibits the Bactericidal Activity of Normal Human Serum
}

\author{
NEAL L. SCHILLER ${ }^{(30)}$ AND RANDAL L. MILLARD \\ Division of Biomedical Sciences and Department of Biology, University of California, Riverside, California, USA
}

\section{Summary}

Sputum samples were obtained from nimeteen cystic fibrosis (CF) patients with respiratory tract infections due to Pseudomonas aeruginosa and were examined for the presence of a specific local protective or blocking factor, which might partially explain the inability of CF patients' pulmonary defense mechanisms to clear their lungs of Pseudomomas infection. Using an assay that measures the bactericidal activity of fresh normal human serum (FHS), eighteen of nineteen CF sputa examined were capable of protecting either autologous or heterologous strains of $P$. aeruginosa from serum bactericidal activity. Most of this protective activity was absorbable at $4^{\circ} \mathrm{C}$ by Pseudomonas, and could be reduced by dilution in phosphate buffered saline or counteracted by an increase in serum concentration. This protective activity is believed to be due to a Pseudomonas-specific bactericidal blocking antibody. In contrast, only two of 17 non-CF sputa were found to significantly inhibit the bactericidal activity of FHS for $P$. aeruginosa. Furthermore, three out of five CF sputa also protected Escherichia coli from the bactericidal activity of FHS. This protective activity was much less than that observed with $P$. aeruginosa, and was not absorbable at $4^{\circ} \mathrm{C}$, suggesting the presence of a second blocking or protective factor in CF sputum. From these observations, we conclude that sputum from CF patients infected with $P$. aeruginosa contains one or more factors that interfere with the bactericidal activity present in FHS.

\section{Abbreviations}

BHI, brain heart infusion

CF, cystic fibrosis

CFU, colony forming unit

FHS, fresh normal human serum

PBS, phosphate buffered saline

PIA, pseudomonas isolation agar

sIgA, secretory IgA
Chronic obstructive pulmonary infection is the major cause of morbidity and mortality in people with cystic fibrosis (CF). Although respiratory infection with Staphylococcus aureus often occurs, Pseudomonas aeruginosa (particularly the mucoid colonial form) eventually emerges as the dominant pathogen in $70-90 \%$ of CF patients, and $>90 \%$ die of progressive pulmonary insufficiency complicated by infection with $P$. aeruginosa (4).

Except for respiratory tract infections, CF children are not more susceptible to infection than normal children of comparable age. $P$. aeruginosa is known to be an opportunistic pathogen, which commonly infects the debilitated patient $(25,26)$, and is seldom isolated from pediatric patients with chronic respiratory disease other than CF $(4,11)$. These basic clinical observations, coupled with the fact that pulmonary infections rarely spread outside the respiratory tract in CF patients, strongly suggest a defect in local pulmonary defense mechanisms.

Despite the presence of elevated levels of Pseudomonas antibodies in CF patients' sera and pulmonary secretions $(13,15)$, these bacteria are not cleared by pulmonary defense mechanisms. Alveolar macrophages isolated from CF patients appear normal (23), but CF serum appears to interfere with the ability of rabbit and human alveolar macrophages to ingest and destroy Pseudomonas $(2,3,5,21,23)$. This effect has been attributed to either a serum deficiency (2), or a serum inhibitory factor $(3,21,23)$. Fick et al. (5) have recently demonstrated that $\mathrm{IgG}$ antibodies from CF patients are impaired in their attachment to the Fc receptors of human alveolar macrophages.

In addition to interfering with phagocytosis, CF serum has also been found to block serum bactericidal activity for $P$. aeruginosa. Whereas $P$. aeruginosa strains isolated from CF patients were more sensitive to the bactericidal activity of normal human serum than strains isolated from non-CF patients (12, and Schiller, N.L., manuscript in preparation), sera from CF patients chronically infected with $P$. aeruginosa showed a selective inability in bactericidal activity against the patient's own $P$. aeruginosa isolate, 